\title{
Ansiedad social, adicción al internet y al cibersexo: su relación con la percepción de salud
}

\author{
Social anxiety, Internet and Cibersex addiction: its relationship with health perception
}

\author{
John Alexander Castro \\ Stefano Vinaccia \\ Fundación Universitaria Sanitas, Colombia \\ Rafael Ballester-Arnal \\ Salusex-Unisexsida. Departamento de Psicología Básica, Clínica y Psicobiología \\ Universitat Jaume I de Castellón, España
}

Rec (28 de marzo de 2018) Acept (5 de noviembre de 2018)

\begin{abstract}
Resumen
Se realizó una investigación cuantitativa, descriptiva, correlacional de corte transversal, para describir la posible relación entre la ansiedad social, la adicción a internet y al cibersexo, con la percepción de bienestar subjetivo y de malestar físico. Participaron 214 adultos jóvenes, con edades entre los 18 y 30 años, de la ciudad de Bogotá, Colombia. Los resultados evidenciaron un nivel bajo en el comportamiento de las variables y relaciones significativas, con diferencias en función del sexo, siendo en las mujeres la relación significativa y positiva entre la adicción al internet y la adición al cibersexo con la percepción de malestar físico, y en los hombres, significativa y negativa entre la adicción al internet y al cibersexo con la percepción de bienestar subjetivo. Se discuten los resultados y sus implicaciones, para el mejoramiento del bienestar y la calidad de vida del adulto joven.

Palabras clave: Adicción al cibersexo, adicción a internet, bienestar subjetivo, malestar físico, jóvenes.
\end{abstract}

\begin{abstract}
A quantitative, descriptive and correlational research was done, with Cross - sectional design, with the aim to describe the possible significant relationship between the social anxiety, internet use addiction and Cybersex addiction, with the perception of subjective well-being and physical discomfort. Participants were 214 young adults, women and men with ages between 18 to 30 years old; all of them from the city of Bogotá, Colombia. The results showed low level measures in all of the variables with significant relationships between them. Also, showed significant differences depending on gender. For women, the relationship was stronger and positive between the internet and cybersex addiction and their perception of physical discomfort, for men was stronger and negative between internet and cybersex addiction and their subjective well-being perception. The results are discussed and its implications, in order to improve the well-being and the quality of life of the young adults.

Keywords: Cybersex addiction, Internet addiction, subjetive well-being, Physical discomfort, young people.
\end{abstract}

Correspondencia: John Alexander Castro Muñoz, address for correspondence: 7th Street - 173 - 64 - Bogotá - Colombia, e-mail johacastro@ unisanitas.edu.co, Telephone: 57 - 3165387869- PBX: 57 - 1- 589537. Stefano Vinaccia Alpi, 7th Street - 173 - 64 - Bogotá - Colombia, email: svinaccia@unisanitas.edu.co.Rafael Ballester-Arnal, Avenida de Vicent Sos Baynat s/n 12071, Catellon, Castello, España, e-mail: rballest@uji.es 


\section{Introducción}

De acuerdo con la revisión de antecedentes sobre la presunta relación entre la ansiedad social, la adicción al internet y la adicción al cibersexo, Montesi et al. (2013) identificaron relaciones significativas entre la ansiedad social y la calidad de vida sexual en jóvenes, con implicaciones negativas para la intimidad y la comunicación. Esto junto con el precedente de Orzack \& Ross (2000) acerca del papel de la baja autoestima y la insatisfacción sexual en el cibersexo. De otro lado, aunque la adicción a internet podría ser una condición asociada al cibersexo, no es la única fuente de varianza del fenómeno. De acuerdo con Young (1999) citado por Ballester et al. (2010), el cibersexo puede expresar la adicción al internet, aunque también podría tratarse de un subtipo de adicción al sexo. Se presume alto el grado de asociación, tesis que validó previamente Davis (2001) y Ortiz-Gómez \& Muñoz-García (2005) quienes consideraron a la ansiedad social y la presencia de otros desórdenes como variables asociadas al uso de patológico de internet. Adicionalmente Armstrong \& Khawaja (2002) consideraron que dicha ansiedad social puede variar significativamente de acuerdo al sexo de las personas. Es por eso que éste, junto con otros antecedentes similares, sustentan la hipótesis principal a partir de la cual se buscó en este estudio evaluar posibles asociaciones entre la ansiedad social, la adicción al internet y el cibersexo, considerando el sexo, tal y como se observa en la figura 1.

Adicionalmente, no se ha considerado suficientemente el abordaje del cibersexo desde una perspectiva que no se centre necesariamente en el déficit y la patología. A partir de ello, en esta investigación se consideró su abordaje desde una perspectiva positiva que contemple su posible relación con la percepción de bienestar subjetivo y de salud física. Con el fin de clarificar estos conceptos a continuación se sintetizan las definiciones y los principales antecedentes que sustentan el problema de esta investigación. En la figura 2 se presenta el modelo final de variables.

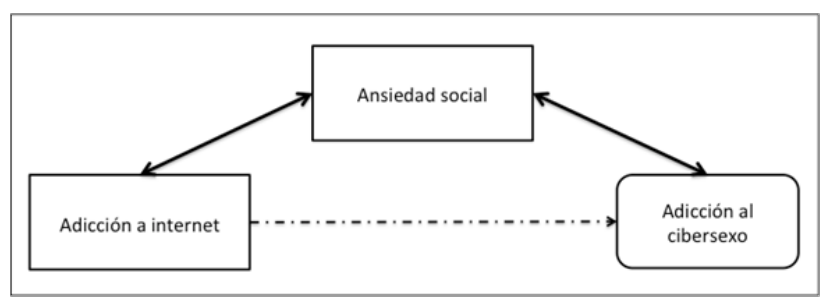

Figura 1. Esquema inicial de relación entre las variables.

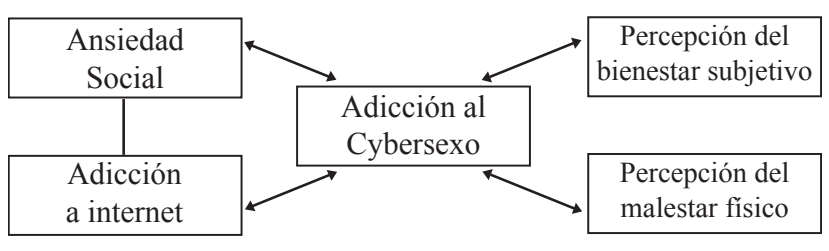

Figura 2. Modelo final de variables

La ansiedad social es un temor generalizado ante la interacción directa con otros, incluye la predisposición a ser fácilmente persuasible y atender a las evaluaciones de los otros. Schlenker \& Leary (1982) mencionaron que surge de imaginar o inferir evaluaciones o impresiones negativas por parte de los otros. Kimbrel (2008) afirma que implica hipersensibilidad a la estimulación, con temor generalizado hacia actuaciones en público, que se evidencia por una alta reactividad de la amígdala, con lo que concuerdan otros autores como Antona, Delgado, García \& Estrada (2012), Kambouroupoulus, Egan, O’Connor, \& Staiger (2014), Labuschagne et al. (2010). Lee \& Stapinski (2012) encontraron que la ansiedad social, por su parte, está asociada con la evaluación negativa de las relaciones interpersonales. Lo que una vez más corresponde con lo formulado desde 2005 por Ortiz-Gómez y Muñoz-García (2005) para el caso del uso patológico de internet.

Respecto al uso de internet al igual que con cualquier adicción, resulta complejo diferenciar entre un uso excesivo o adictivo. Para Beranuy, Chamarro, Graner \& Carbonel (2009) corresponde a una adicción si concuerda con los criterios del juego patológico, o de otras adicciones conductuales o toxicas, incluyendo en este caso específico el "deseo de estar conectado".

La adicción al cibersexo proviene de la clasificación de Cooper \& Griffin-Shelley (2002) y en la que coincide la revisión realizada por Wéry \& Billieux (2015), que la define como uso excesivo de internet con fines sexuales, lo que incluye el acceso a contenido pornográfico, interacción con otros usuarios incluyendo el uso de webcam, establecer contacto con terceros y acceder a realidades virtuales con el mismo propósito. Definición que concuerda con lo referido por Beranuy et al. (2009), en la que se resalta que desde esta perspectiva implica afecciones negativas en las áreas de ajuste. En 2003 Cooper et al identificaron que es mayor en los hombres. Ballester, Gil, Gómez \& Gil (2010) han expuesto que la adicción al cibersexo es una patología de rápido crecimiento, y con ese fin han adaptado y validado en población hispana la escala Internet Sex Screening Test (ISST) creada por Delmonico (1997) y revisada nuevamente por Delmonico y Miller (2003), para su evaluación. 
¿Es el Cibersexo una adicción? Existen dos posturas, una orientada a los "beneficios" del consumo de pornografía o de interacción en realidades sexuales virtuales (Laier, Powlikowski, Pekal, Schulte, \& Brand, 2013); la otra, resalta las consecuencias negativas a nivel psicológico, social y físico (Dorton \& Cast, 2007), en la que el centro de análisis es su influencia negativa sobre las áreas de ajuste.

Respecto al consumo frecuente de pornografía, o de interacción sexual virtual con otros, Dorton \& Cast (2007) aseguran que los efectos son negativos al fomentar el sexo individual compulsivo, siendo los adolescentes y adultos jóvenes el grupo de mayor riesgo. Philaretou, Mahfouz \& Allen (2005) encontraron en hombres relaciones significativas y positivas entre el consumo de pornografía, la ansiedad generalizada y la depresión, y negativas con la satisfacción en relaciones íntimas directas, al idealizar los referentes pornográficos. Dichos ideales, se transfieren, como búsqueda de similitud con las estructuras mesomórficas de los referentes del material, con efectos negativos sobre el afecto positivo, generando apego inseguro en las relaciones de pareja (Tylka, 2015). Behm-Morawitz (2013) encontró, al estudiar los "reflejos del sí mismo" refiriéndose al uso de un Avatar o su equivalente, que dichas representaciones afectan la apariencia física, con implicaciones negativas en el bienestar subjetivo.

Laier et al. (2013) evaluaron y contrastaron las diferencias entre la excitación que se produce por material pornográfico, y aquella que no, encontrando mayor la gratificación procedente del material pornográfico que se mantiene en el tiempo. Finalmente, Schiebener, Laier \& Brand (2015) concluyeron que, como consecuencia de la adicción al cibersexo, se reduce la capacidad para atender múltiples demandas y orientar el trabajo al cumplimiento.

Desde una perspectiva de género, existe evidencia de mayores niveles de ansiedad social en mujeres (Crimmins, Kim \& Sole-Auro, 2011; McLean, Asnaani, Litz \& Hofmann, 2011); igualmente mayor preocupación en ellas por la salud física y mental (Roberts, Hodgson \& Dolan, 2011). Al respecto aclaran Stewart, Taylor \& Baker (1997) que en las mujeres hay más preocupación por la salud física y en los hombres, por la salud psicológica. Para Rosenfield, \& Mouzon (2012), esto se explicaría por la asociación entre la condición biológica y la construcción social que "induce", al parecer en el contexto sociocultural en el que se desarrolló la investigación, al fomento de concepciones de masculinidad y feminidad en las cuales, en las mujeres el riesgo se asocia con la internalización de los problemas y en los hombres con la externalización. A partir de esto se podría inferir que en las mujeres se desarrollen mejores estrategias de afrontamiento caracterizadas por conductas internalizantes y en los hombres caracterizadas por conductas externalizantes, desarrollando por lo tanto en cada grupo mayores niveles de ansiedad en los dominios donde no se desarrollan dichas estrategias. Son similares los hallazgos de Eaton et al. (2012) que sustentan esta inferencia.

En esta investigación se evalúa la relación entre la ansiedad social, la adicción a internet y la adicción al cibersexo, con la percepción de bienestar subjetivo de acuerdo a Diener (1985 entendida como el grado de satisfacción generalizado con la vida) y con la percepción de malestar físico a partir de Derogatis (1994 como la percepción generalizada de funcionalidad), con el propósito de establecer si el cibersexo puede estar relacionado, desde la perspectiva del sujeto, con la percepción de su salud.

\section{Objetivo de la investigación}

El objetivo general es observar y describir si la ansiedad social y la adicción al internet se relacionan con la adicción al cibersexo, y si éste, a su vez, se relaciona con la percepción de bienestar subjetivo y malestar físico como consecuencia de dicha adicción, para el grupo de hombres y de mujeres de manera independiente. Para ello, se requirió considerar el comportamiento de las variables en cada grupo de acuerdo al sexo y determinar el tipo de relaciones en cada uno de estos grupos

De acuerdo con los antecedentes revisados, se consideró como hipótesis general de la investigación la existencia de relaciones significativas entre la ansiedad social, la adicción al internet y al Cibersexo, y de éstas a su vez con las percepciones de bienestar subjetivo y de malestar físico. Finalmente se consideró que tanto el comportamiento de las variables como las relaciones entre éstas podría variar en su fuerza de asociación, nivel de significación y dirección de las asociaciones, en función del sexo de los participantes.

\section{Método}

\section{Diseño de investigación}

Cuantitativa, descriptiva, con metodología correlacional, de corte transversal

\section{Participantes}

La muestra estuvo conformada por 214 adultos jóvenes de Bogotá (Colombia) con edades entre los 18 y 30 años, con una media de 21,71 y desviación estándar de 3,43 de ambos sexos - 137 mujeres y 77 hombres-, de niveles socioeconómicos alto, medio y bajo. La orientación sexual se evaluó con la estrategia sugerida del análisis propuesto por Booker (2012) a partir de sus análisis a las aproximaciones conceptuales de Savin-Williams, y considerando así 
mismo las sugerencias de Kinsey referenciadas inicialmente en los análisis de Storms (1980) donde los participantes en un continuo que va desde 1 "Homosexual" hasta 10 "Heterosexual" se clasifican. Se estableció como punto de corte 5,5 , siendo así por debajo de este valor "Más homosexual" por encima sin incluir este valor "Más heterosexual". De acuerdo con esta estrategia, el 18\% (39 participantes) se clasificó como "más homosexual" y el 82\% (175 participantes) como "más heterosexual".

\section{Instrumentos}

\section{Ansiedad social}

Se empleó la adaptación al español del Social Interaction Anxiety Scale SIAS-20 de Mattick \& Clarke (1998), traducida y adaptada en México por Moral, García y Antona (2013). Está compuesta por 20 ítems que se responden en una escala tipo Likert de cinco puntos, de 0 a 4 , donde 0 significa "nada" y 4 "totalmente". El valor Alfa de Cronbach de la versión adaptada fue de 0,87 , y para esta aplicación fue de 0,79 .

\section{Adicción a internet}

Se empleó el Cuestionario de Problemas Relacionados con Internet-PRI, construido por De García, Vigo, Fernández \& Marco (2002), a partir de ítems seleccionados de los cuestionarios IRABI (Internet Related Adictive Behavior Inventory) de Brenner (1997) y IRPS (Internet Related Problem Scale) de Amstrong, Phillips \& Saling (2000), basadas en el IAT - Internet Addiction Test de Goldberg (1996), referenciado en Panayides \& Walker (2012). Esta versión está conformada por 10 ítems que se responden en una escala tipo likert de 5 puntos la cual va de 1 que significa "Nunca" a 5 "Siempre". El valor del Alfa de Cronbach obtenido en la versión de validación fue de 0,71 , y para esta aplicación fue de 0,89 .

\section{Adicción al Cibersexo}

Se empleó el cuestionario adaptado y validado por Ballester et al., (2010) Cuestionario de adicción al Cibersexo, adaptado del Internet Sex Screening Test de Delmónico (1997), revisado por Delmónico \& Miller (2003) y validado y con puntuaciones normalizadas por Carnes, Delmónico \& Griffin (2007). Consta de 25 ítems que se responden en una escala dicotómica de verdadero/falso. El valor del alfa de Cronbach en su validación fue de 0,88 , y para esta aplicación fue de 0,91

\section{Bienestar subjetivo}

Se empleó la escala de percepción de satisfacción con la vida - Satisfaction With Life Scale (SWLS) de Diener, Emmons, Larsen \& Griffin (1985) en su versión oficial, referenciada en Pavot \& Diener (2008), a partir de lo estipulado por el autor, y por las sugerencias consignadas para su aplicación, en Kobau, Sniezek, Zack, Lucas \& Burns (2010). Esta consta de 5 ítems que se responden en una escala tipo Likert de 7 puntos que va desde "completamente en desacuerdo" hasta "completamente de acuerdo". El alfa de Cronbach reportado en la literatura es de 0,79. Para esta aplicación fue de 0,77 .

\section{Percepción de malestar físico}

Se empleó el Inventario de síntomas Symptom Cheklist SCL-90 de Derogatis (1994) en la versión citada, traducida y adaptada para México por Blas, González, Chávez, Cruz \& López (2006). Consta de 90 ítems que evalúan en una escala tipo Likert de cinco puntos que va de "nada" a "mucho", indicadores generales de percepción de malestar en 9 dimensiones: somatizaciones, obsesiones y compulsiones, sensibilidad interpersonal, depresión, ansiedad, hostilidad, ansiedad fóbica, ideación paranoide y psicoticismo. Para esta investigación se empleó la dimensión de somatización, que evalúa la percepción de malestar físico a partir de las percepciones de malestar o estrés que reflejan disfunción corporal. Está conformada por 11 ítems. En la versión validada para España, la puntuación de alfa de Cronbach para esta dimensión fue de 0,85 , y para esta investigación fue de 0,83 .

\section{Procedimiento}

La aplicación se hizo en un periodo de 3 meses. El muestreo fue a conveniencia bajo la estrategia de "bola de nieve". Se siguieron las mismas instrucciones de aplicación por parte de los investigadores, auxiliares y estudiantes del semillero de investigación en "Cognición social y bienestar" de la Fundación Universitaria Sanitas. Posteriormente se realizaron los análisis estadísticos. 


\section{Resultados}

\section{Análisis descriptivos}

Se calcularon los valores de la media y desviación estándar para cada una de las variables. En la tabla 1 se presentan estos resultados.

Por lo que respecta a la ansiedad social, de acuerdo al cuestionario empleado en el que no se establecen con precisión puntos de corte, las puntuaciones por encima de la media, que por lo tanto se considerarían como puntuaciones clínicas, es decir con superiores a 40 en el rango de 0 a 80 , se obtuvieron en el 13,1\%, de los participantes. La adicción al internet evidenció una media próxima al criterio "sin problemas". El porcentaje de participantes con puntuaciones clínicas o con "problemas ocasionales" (valores superiores a 45) fue de 9,8\%. Para el caso de "problemas frecuentes" (valores superiores a 65), no se presentaron casos. En cuanto a la adicción al cibersexo, se observaron puntuaciones en el nivel denominado como "usuarios en riesgo" en el 13\% de los participantes, y en un $1 \%$ "usuarios patológicos".
Respecto a la percepción de bienestar psicológico, únicamente el 19,2\% de los participantes obtuvo puntuaciones que reflejan un nivel bajo de satisfacción con la vida. Finalmente, respecto a la percepción de malestar corporal, en un $36 \%$ de la muestra, se observaron puntuaciones que reflejan dificultades.

\section{Análisis diferenciales en función de la orientación sexual y el género}

El análisis de normalidad de los datos se hizo a través de la prueba no paramétrica de Kolmogorov-Smirnov. De acuerdo con el resultado, las variables no cumplieron con el supuesto de normalidad; por lo tanto, los análisis diferenciales se realizaron mediante estadísticos no paramétricos.

Inicialmente se compararon a través del análisis estadístico de la U de Mann-Withney para determinar si había diferencias significativas en función de la orientación sexual. Los resultados se presentan en la tabla 2 y evidencian la ausencia de diferencias significativas en ninguna de las variables

Tabla 1. Descriptivos de las variables de la investigación.

\begin{tabular}{|c|c|c|c|c|c|}
\hline & Mínimo & Máximo & Media & $\begin{array}{l}\text { Desviación } \\
\text { estandar }\end{array}$ & Baremos - Criterios de estimación \\
\hline $\begin{array}{l}\text { Ansiedad } \\
\text { Social }\end{array}$ & 8 & 61 & 28,50 & 10,18 & $\begin{array}{l}\text { De acuerdo con Bögels et al ( } 2010 \text {, citado por Moral, García } \\
\text { y Antona, (2013), al evaluarse este constructo a partir de los } \\
\text { criterios de la fobia social, como característica desdaptativa, } \\
\text { se espera que su distribución sea asimétrica positiva, con } \\
\text { valores bajos. }\end{array}$ \\
\hline $\begin{array}{l}\text { Adicción a } \\
\text { Internet }\end{array}$ & 19 & 58 & 30,21 & 8,90 & $\begin{array}{l}\text { El comportamiento de las puntuaciones se estableció por } \\
\text { parte de los autores mediante un análisis clúster de tres } \\
\text { grupos con los siguientes valores para las puntuaciones } \\
\text { medias totales: } \\
\text { Sin problemas: } \mathrm{M}=26,27 \\
\text { Problemas ocasionales: } \mathrm{M}=45,16 \\
\text { Con problemas frecuentes: } \mathrm{M}=65,35\end{array}$ \\
\hline $\begin{array}{l}\text { Adicción al } \\
\text { Cibersexo }\end{array}$ & 0 & 25 & 4,19 & 5,08 & $\begin{array}{l}\text { De acuerdo con Carnes, Delmónico \& Griffin (2007) las } \\
\text { puntuaciones permiten una clasificación de tres niveles: } \\
\text { Usuarios recreativos }(0-8) \\
\text { Usuarios en riesgo }(9-18) \\
\text { Usuarios patológicos }(19-25)\end{array}$ \\
\hline $\begin{array}{l}\text { Percepción } \\
\text { de bienestar } \\
\text { subjetivo }\end{array}$ & 8 & 35 & 24,03 & 5,38 & $\begin{array}{l}\text { Criterios establecidos para los valores de la media: } \\
\text { Entre } 5 \text { y 9: Extremadamente insatisfecho } \\
\text { Entre } 10 \text { y 14: Insatisfecho } \\
\text { Entre } 15 \text { y 19: Por debajo del promedio de satisfacción } \\
\text { Entre } 20 \text { y 24: Satisfacción promedio } \\
\text { Entre } 25 \text { y 29: Satisfacción alta } \\
\text { Entre } 30 \text { y 35: Altamente satisfecho con la vida }\end{array}$ \\
\hline $\begin{array}{l}\text { Percepción } \\
\text { de malestar } \\
\text { físico }\end{array}$ & 0 & 84 & 13,42 & 10,30 & $\begin{array}{l}\text { La puntuación media de este factor en la validación } \\
\text { mexicana fue de } 8,76 \text {. El instrumento, al estar diseñado } \\
\text { para la evaluación con puntuaciones promedio en pacientes } \\
\text { psiquiátricos, se esperaría que las puntuaciones fueran bajas. }\end{array}$ \\
\hline
\end{tabular}


Tabla 2. Diferencias de medias de acuerdo a la orientación sexual de los participantes

\begin{tabular}{lllll}
\hline Variable & $\begin{array}{l}\text { Clasificación de la } \\
\text { orientación sexual }\end{array}$ & Media & $\begin{array}{l}\text { Desviación } \\
\text { Estándar }\end{array}$ & $\begin{array}{l}\text { Valor P de la prueba U } \\
\text { de Mann - Whitney }\end{array}$ \\
\hline Ansiedad social & Más homosexual & 28,92 & 12,64 & 0,99 \\
Adicción a internet & Más heterosexual & 28,40 & 9,58 & \\
& Más homosexual & 29,79 & 9,01 & 0,68 \\
Adicción al Cibersexo & Más heterosexual & 30,30 & 8,89 & 0,98 \\
\multirow{2}{*}{ Bienestar subjetivo } & Más homosexual & 4,15 & 5,08 & \\
& Más heterosexual & 4,19 & 5,09 & 0,67 \\
Malestar físico & Más homosexual & 24,35 & 5,07 & \multirow{2}{*}{0,75} \\
& Más heterosexual & 23,95 & 5,45 & \\
& Más homosexual & 14,17 & 10,86 & \\
\hline
\end{tabular}

Con respecto al género, se encontraron diferencias significativas sólo para la adicción al cibersexo, mayor en los hombres, y para la percepción de malestar físico, mayor en las mujeres. Esta comparación por género se presenta en la tabla 3.

Tabla 3. Diferencias en función del sexo

\begin{tabular}{lllll}
\hline Variable & Sexo & Media & $\begin{array}{l}\text { Desviación } \\
\text { Estándar }\end{array}$ & $\begin{array}{l}\text { Valor P de la prueba U } \\
\text { de Mann - Whitney }\end{array}$ \\
\hline Ansiedad social & Mujer & 28,64 & 10,45 & 0,93 \\
Adicción a internet & Hombre & 28,26 & 9,74 & \\
& Mujer & 29,53 & 8,57 & 0,12 \\
Adicción al Cibersexo & Hombre & 31,42 & 9,40 & \\
\multirow{2}{*}{ Bienestar subjetivo } & Mujer & 3,40 & 5,02 & 0,00 \\
& Hombre & 5,58 & 4,93 & \\
Malestar físico & Mujer & 24,03 & 5,24 & 0,86 \\
& Hombre & 24,03 & 5,66 & \\
& Mujer & 14,69 & 11,40 & 0,03 \\
\hline
\end{tabular}

Relación entre las distintas variables y capacidad predictiva de las mismas

A continuación se presentan los resultados del análisis de correlación para toda la muestra en la tabla 4.

La adicción al Cibersexo se relaciona significativa y positivamente con la ansiedad social, la adicción al internet y la percepción de malestar físico, y negativamente con la percepción de bienestar subjetivo. La relación entre el bienestar subjetivo y el malestar corporal / físico es significativa y negativa.

Posteriormente se realizaron los análisis de regresión lineal para todos los participantes. Respecto al posible papel predictivo de la adicción al internet y la ansiedad social sobre el Cibersexo, se observa que únicamente la adicción al internet predice la presencia de adicción al Cibersexo, con un valor del beta de $0,46, p<0,01$ y $R$ cuadrado ajustado de 0,22 . En la interacción de la adicción a internet con la ansiedad social, no se obtiene significación estadística.

A partir de este resultado, se realizó el mismo análisis, con la percepción de bienestar subjetivo como variable dependiente, con la adicción al internet y a Cibersexo como posibles predictoras. En este caso se observó que ambas variables predicen la percepción de bienestar subjetivo. 
Tabla 4. Análisis de correlación de Spearman para todos los participantes

\begin{tabular}{llllll}
\hline & 1 & 2 & 3 & 4 & 5 \\
\hline 1. Ansiedad social & & & & & \\
2. Adicción a internet & $0,52^{* *}$ & & & & \\
3. Adicción al Cibersexo & $0,25^{* *}$ & $0,41^{* *}$ & & & \\
4. Bienestar subjetivo & $-0,18^{* *}$ & $-0,22^{* *}$ & $-0,19^{* *}$ & & \\
5. Malestar físico & $0,26^{* *}$ & $0,26^{* *}$ & $0,21^{* *}$ & $-0,22^{* *}$ & \\
\hline
\end{tabular}

*La correlación es significativa con valor $\mathrm{p}<0,05$

**La correlación es significativa con valor $\mathrm{p}<0,01$

En el primer paso, con la adicción a internet como única predictora, se obtuvo un valor beta de $-0,16$, con un valor $\mathrm{p}<0,05$ y un $\mathrm{R}$ cuadrado ajustado de 0,05 . Al ingresar la interacción entre la adicción al internet y al cibersexo como predictoras, los valores apenas evidenciaron una mejor capacidad predictiva con un valor Beta de $-0,17$, con un valor $\mathrm{p}<0,05$ y un $R$ cuadrado ajustado de 0,07 .

El mismo análisis se realizó con la percepción de malestar físico como variable dependiente. En este caso, se observó únicamente a la adicción al internet como predictora, con un valor Beta de 0,14 , valor $\mathrm{p}<0,05$ y un $\mathrm{R}$ ajustado de 0,028 . Al ingresar la interacción entre la adicción al internet y la adicción al cibersexo, los valores no variaron significativamente, representando incluso una reducción en su capacidad predictiva.

De conformidad con los objetivos e hipótesis de investigación, se realizaron los análisis de correlación y regresión por separado para mujeres y hombres

En el grupo de las mujeres, se observaron relaciones significativas entre las variables con excepción del bienestar subjetivo, que sólo evidenció relaciones significativas y negativas con la adicción a internet. De acuerdo con este resultado, en el grupo de las mujeres se realizaron análisis de regresión lineal, a partir de dos modelos. El primero, con la adicción al cibersexo como dependiente de la ansiedad social y la adicción al internet. El segundo, con la percepción de malestar físico como dependiente. Respecto al primer modelo los resultados evidenciaron una alta capacidad predictiva, de la adicción al internet sobre la adicción al cibersexo. Beta $=0,43, \mathrm{p}<0,01$ y $\mathrm{R}$ cuadrado ajustado de 0,21 . Este resultado se vio afectado al introducir la interacción entre la adicción al internet y la ansiedad social como predictivas con un valor Beta de 0,08 , un nivel de significación con un valor $\mathrm{P}$ superior a 0,05 en el que por lo tanto no se cumple con el criterio, con un valor del R cuadrado ajustado de 0,21. En las mujeres la adicción al internet es la variable que mejor predice la adicción al cibersexo. Es, asímismo, la variable que en este grupo, mejor predice la percepción de malestar físico con un valor Beta de 0,17, $\mathrm{p}<0,05$ y $\mathrm{R}$ cuadrado ajustado de 0,037 .

Para los hombres el análisis de correlación evidenció relaciones significativas entre todas las variables del modelo, menos con la percepción de malestar físico.

En este grupo al introducir las variables en el modelo de regresión lineal con la adicción al cibersexo como dependiente, se observó que únicamente la adicción al cibersexo se relaciona significativamente con la percepción de malestar físico como independiente, con un valor beta de 0,41 , $\mathrm{p}<0,01$ y un $\mathrm{R}$ cuadrado ajustado de 0,18 .

Dentro del segundo modelo, con el bienestar subjetivo como dependiente de la ansiedad social y la adicción al cibersexo, al introducir en el análisis las dos predictoras, los resultados arrojaron en la interacción un valor Beta de $-0,21, p<0,01$ y un $R$ cuadrado ajustado de 0,06 .

\section{Discusión}

De acuerdo con las hipótesis se resaltan varios hallazgos y sus implicaciones, enfatizando específicamente: las diferencias por género, las relaciones significativas en toda la muestra y las diferencias en las relaciones, en función del género.

Respecto a las puntuaciones en las principales variables, éstas se encuentran en niveles bajos, como en el caso de la adicción al internet, pese a la alta disponibilidad de acceso. Al parecer las respuestas de los participantes, parcialmente, podrían responder a la deseabilidad social. Algo similar ocurre con la adicción al cibersexo. Se sugieren futuras investigaciones, con población que en su mayoría alcance el criterio sujetos problemáticos de adicción al cibersexo, como usuarios "en riesgo" o "patológicos". Por otra parte la prevalencia de adicción al internet del $9,8 \%$ no resulta del todo coherente con el análisis que en 2017 realizan en otros contextos Dau, Hoffmann \& Banger, quienes afirman que debería corresponder con la proporción en que se 
considera importante su uso, con un $89 \%$ de los hombres y un $86 \%$ de las mujeres que así lo consideran. Esto podría entre otras estar explicado por la falta de consenso sobre la operacionalización del uso de internet y la consideración de éste como adicción, acorde a lo que resaltan Kuss \& López-Fernandez (2016), y Starcevic \& Aboujaoude (2017) con miras a establecer criterios claros de intervención. Aun así, se debe hacer una lectura conjunta entre la prevalencia y la prevalencia de los factores asociados, en este caso de la ansiedad social, que podría explicar el bajo nivel de prevalencia, tal y como lo sugiere en su análisis Brand (2017).

Sobre la asociación entre las variables, los resultados confirman algunas de las hipótesis. Específicamente en la regresión lineal con toda la muestra, la ansiedad social como predictora de la adicción al cibersexo, así como del bienestar subjetivo y malestar físico, aunque con una mayor capacidad predictiva por parte de la adicción al internet.

Acerca del cibersexo, los hallazgos sugieren una cierta capacidad predictiva sobre éste, de la adicción al internet, particularmente en los hombres. Este dato habla de una posible comorbilidad entre las adicciones. Esto es coherente con lo que sugieren Beranuy et al. (2009), así como Starcevic \& Aboujaoude (2017) al considerar el uso de internet como adicción, cuando altera las áreas de ajuste, y que según Cia (2013), coincide con los criterios del juego patológico. En concordancia, Cooper \& Griffin-Shelley (2002) y Ballester et al. (2010) afirman que pueda considerársele como una adicción conductual y que quizás entre las razones, del paso del uso funcional o saludable al uso adictivo, se encuentre el papel de la adicción al internet, entre otras variables. Este hallazgo también coincide con Schiebener Laier \& Brand (2015) que afirman que el cibersexo es adictivo en la medida en que interfiere con el cumplimiento de compromisos, como en el caso de la adicción al internet. Aunque no existen explicaciones claras para comprender el paso de un consumo saludable a uno adictivo, varias hipótesis se han formulado. Entre ellas, Grov et al, (2008) argumenta que la pérdida del control podría estar asociada a la aparición de estados emocionales negativos en ausencia del uso, el aumento de la tolerancia y la abstinencia. De otro lado, se encuentra la hipótesis según la cual podría tratarse de una manifestación de la relación del cibersexo adictivo con la adicción al internet y la adición al sexo (Griffits, 2012; Wéry \& Billieux, 2015).

La relación encontrada entre la ansiedad social y el Cibersexo corrobora lo propuesto previamente en la revisión teórica de Ortiz-Gómez y Muñoz-García (2005) y los hallazgos de Caplan (2007) que, junto con otros indicadores de desajuste o malestar, consideraban que podría predecir el uso patológico de internet. Resultando adicionalmente coherente con la conceptualización de la ansiedad social que considera la mayor sensibilidad y consecuente sobreestimu- lación por parte de quien la padece, a las interacciones sociales en las que no media el uso de la interacción a distancia como en el caso de aquellas que propicia internet (Antona et al. 2012; Hedman et al., 2010; Kimbrel, 2008; Lee \& Stapinski, 2012; Sears, 1967; Watson \& Friend; 1969). En esta misma dirección, los hallazgos de la investigación realizada por Tan (2018) sobre la relación entre el apoyo social y la adicción a internet, validan este resultado al encontrar una relación significativa entre apoyo social y adicción al internet, donde la percepción de apoyo social aumenta con el contacto personal.

Desde la psicología de la salud, acorde con McLean et al. (2011), Crimmins et al. (2011) y Roberts et al. (2011) las mujeres evidencian mayor preocupación por la salud física. Esto es coherente con Stewart, Taylor \& Baker (1997) y reafirmado por Eaton et al. (2012), sobre una mayor preocupación hacia indicadores de salud próximos a las conductas internalizantes y en los hombres hacia las conductas externalizantes. Estas diferencias reafirman lo considerado por Rosenfield \& Mouzon (2012) para quienes resultan de las construcciones sociales de feminidad y masculinidad, que conllevan a un mayor desarrollo del afrontamiento en esos dominios, por lo que la ansiedad social se relaciona significativamente con los indicadores de salud en los ámbitos opuestos para cada sexo. Sin embargo, se debe considerar que las nociones de bienestar subjetivo provienen de la psicología positiva, mientras que el malestar físico, de la mirada tradicional, más orientada a la enfermedad (Vazquez, Hervas, Rahona \& Gómez, 2009).

Finalmente, es posible afirmar que la hipótesis se confirma, siendo mayor la capacidad predictiva de la adicción al internet sobre la adicción al cibersexo fortaleciendo la idea de una comorbilidad entre los dos trastornos. Las diferencias por sexo reflejan quizás diferencias en las estrategias de afrontamiento. Se sugieren investigaciones que profundicen en la forma como en los hombres la adicción al cibersexo y la ansiedad social predicen la percepción de malestar físico y subjetivo.

\section{Referencias}

Altemus, M., Sarvaiya, N., \& Neill, E. C. (2014). Sex differences in anxiety and depression clinical perspectives. Frontiers in Neuroendocrinology, 35, 320-330. doi: 10.1016/j.yfrne.2014.05.004

Amstrong, L., Phillips, J. G., \& Saling, L. L. (2000). Potential determinants of heavier internet usage. International Journal of Human-Computer Studies, 53, 537-550. doi: 10.1006/ijhc.2000.0400

Antona, C., Delgado, M., García, L., \& Estrada, B. (2012) Adaptación transcultural de un tratamiento para la fobia social: Un estudio piloto. International Journal of Psychology and Psychological Therapy, $12,35-48$. 
Armstrong, K. A., \& Khawaja, N. G. (2002). Gender differences in anxiety: an investigation of the symptoms, cognitions, and sensitivity towards anxiety in a nonclinical population. Behavioural and Cognitive Psychotherapy, 30, 2, 227-231. doi: 10.1017/S1352465802002114

Ballester, R., Gil, M., Gómez S. \& Gil B. (2010) Propiedades psicométricas de un instrumento de evaluación de la adicción al cibersexo. Psicothema, 22, $1048-1053$

Behm-Morawitz, E. (2013) Mirrored Selves: The influence of Self-Presence in a Virtual World on Health, Appearance and Well-Being. Computers in human Behavior, 29, 119 - 128. doi: 10.1016/j.chb.2012.07.023

Beranuy, M., Chamarro, A., Graner., C. \& Carbonel, X. (2009) Validación de dos escalas breves para evaluar la adicción a Internet y el abuso de móvil. Psicothema, 21, 480-485.

Blas, C., González, L., Chávez, R., Cruz, C. \& López L. (2006). Datos sobre la validez y confiabilidad de la Symptom Check List 90 (SCL90) en una muestra de sujetos mexicanos. Salud Mental, 28, 72-81.

Booker, K. (2012). New Studies Offer Clearer Picture of Human Sexuality. Human Ecology, 40(2), 9.

Brand M. (2017) Theoretical Models of the Development and Maintenance of Internet Addiction. In Montag C., Reuter M. (eds) Internet Addiction. Studies in Neuroscience, Psychology and Behavioral Economics. Springer, Cham

Brenner, V. (1997). Psychology of computer use: Parameters of Internet use, abuse and addiction: the first 90 days of the internet usage survey. Psychological Reports, 80, 879-882. doi: 10.2466/pr0.1997.80.3.879

Caplan, S. (2007). Relations Among Loneliness, Social Anxiety, and Problematic Internet Use. Cyberpsychology \& Behavior, 10, 234 - 242. http://dx.doi.org/10.1089/cpb.2006.9963

Carnes, P. J., Delmonico, D. L., \& Griffin, E. (2007). In the shadows of the net: Breaking free of compulsive online sexual behavior (2nd edition). Center City: MN: Hazelden Publishing.

Cia, A. (2013) Las adicciones no relacionadas a sustancias (DSM-5, APA, 2013): un primer paso hacia la inclusión de las Adicciones Conductuales en las clasificaciones categoriales vigentes. Revista de Neuropsiquiatría, 76, 210 - 217. doi: 10.20453/rnp.v76i4.1169

Cooper, A., \& Griffin-Shelley, E. (2002). A quick tour of online sexuality: Part 1. Annals of the American Psychotherapy Association, 5, 11-13.

Courtenay, W. H. (2000). Constructions of masculinity and their influence on men's well- being: a theory of gender and health. Social Science \& Medicine, 50, 1385-1401. doi: 10.1016/S0277-9536(99)00390-1

Crimmins, E. M., Kim, J. K., \& Sole-Auro, A. (2011). Gender differences in health: results from SHARE, ELSA and HRS. The European Journal of Public Health, 21, 81-91. doi: 10.1093/eurpub/ckq022

Davis, R.A. (2001). A cognitive-behavioral model of pathological Internet use. Computers in Human Behavior, 17: 187- 195

Dau W., Hoffmann J.D.G., \& Banger M. (2017) Therapeutic Interventions for Treatment of Adolescent Internet Addiction-Experiences from Germany. In: Montag C., Reuter M. (eds) Internet Addiction. Studies in Neuroscience, Psychology and Behavioral Economics. Springer, Cham

De García, M., Vigo, M., Fernández, M., \& Marco, M. (2002) Problemas conductuales relacionados con el uso de internet: un estudio exploratorio. Anales de Psicología, 18, 273 - 292.
Delmonico, D. L. (1997). Cybersex: High tech sex addiction. Sexual Addiction \& Compulsivity: The Journal of Treatment and Prevention, 4(2), 159-167.

Delmonico, D. \& Miller, J. (2003). The Internet Sex Screening Test: A comparison of sexual compulsives versus non-sexual compulsives. Sexual and Relationship Therapy, 18(3), 261-276. doi:10.1080/1468199031000153900

Derogatis, L. R. (1994). SCL-90-R: Symptom checklist-90-R: administration, scoring \& procedures manual. Minneapolis, Minn: National Computer Systems, Inc.

Diener, E. D., Emmons, R. A., Larsen, R. J \& Griffin, S. (1985). The Satisfaction With Life Scale. Journal of Personality Assessment, 49, 71-75.

Dorton, D. \& Cast, J. (2007) Cybersex Use and Abuse: Implications for Health Education. American Journal of Health Education, 38, 34-40. doi: $10.1080 / 19325037.2007 .10598940$

Eaton, N., Keyes, K., Krueger, R., Balsis, S., Skodol, A., Markon, K., Grant, B. \& Hasin, D. (2012). An invariant dimensional liability model of gender differences in mental disorder prevalence: Evidence from a national sample. Journal of Abnormal Psychology, 121, 282-288. doi: $10.1037 / \mathrm{a} 0024780$

Fergus, T., Kim, H., Valentier, D. \& McGrath, P. (2014) The Social Interaction Anxiety Scale (SIAS) and the Social Phobia Scale (SPS): A Comparison of Two Short-Form Versions. Psychological Assessment, 26, 1281 - 1291. doi: 10.1037/a0037313

Goldberg, I. (1996). Internet addiction disorder. Retrieved from http:// users.rider.edu/ suler/psycyber/supportgp.html

Griffiths, M.D. (2012). Internet sex addiction: A review of empirical research. Addiction Research and Theory, 20, 111-124. http://dx.doi. org/10.3109/16066359.2011.588351

Grov, C., Bamonte, A., Fuentes, A., Parsons, J.T., Bimbi, D.S. \& Morgenstern, J. (2008). Exploring the Internet's role in sexual compulsivity and out of control sexual thoughts/behaviour: A qualitative study of gay and bisexual men in New York City. Culture, Health and Sexuality, 10, 107-124. http://dx.doi.org/10.1080/13691050701564678.

Hedman, E., Ljótsson, B., Rück, C., Furmark, T., Carlbring, P., Lindefors, N., \& Andersson, G. (2010). Internet administration of self-report measures commonly used in research on social anxiety disorder: A psychometric evaluation. Computers in Human Behavior, 26(4), 736-740. doi:10.1016/j.chb.2010.01.010

Kambouroupoulus, N., Egan, S., O'Connor, E. \& Staiger, P. (2014) Escaping threat: Understanding the Importance of Threat Sensitivity in Social Anxiety. Journal of Individual Differences, 35, 47 - 53. doi: 10.1027/1614-0001/a000126

Kimbrel, N. (2008) A model of the development and maintenance of generalized social phobia. Clinical Psychology Review, 28, 592 - 612. doi: 10.1016/j.cpr.2007.08.003

Kobau, R., Sniezek, J., Zack, M. M., Lucas, R. E. \& Burns, A. (2010). Well-being assessment: An evaluation of well-being scales for public health and population estimates of well-being among US adults. Applied Psychology: Health and Well- being, 2, 272-297. doi: 10.1111/j.17580854.2010.01035.x

Kuss, D. J. \& Lopez-Fernandez, O. (2016). Internet addiction and problematic Internet use: A systematic review of clinical research. World 
Journal of Psychiatry, 6, 1, 143-76. doi: 10.5498/jwp.v6.i11.143

Labuschagne, I., Phan, K., Wood A., Angstadt, M., Chua, P., Heinrichs, M., Stout, J. \& Nathan, P. (2010) Oxytocin Attenuates Amygdala Reactivity to Fear and Generalized Social Anxiety Dissorder. Neuropsychopharmacology, 35, 2403 - 2413. doi: 10.1038/npp.2010.123

Laier, C., Powlikowski, M., Pekal, J., Schulte, F. \& Brand, M. (2013) Cybersex addiction: Experienced sexual arousal when watching pornography and not real-life sexual contacts makes the difference. Journal of Behavioral Addictions, 2, 100 - 107. doi: 10.1556/JBA.2.2013.002

Lee, B. \& Stapinski, L. (2012) Seeking safety on the internet: Relationship between social anxiety and problematic internet use. Journal of Anxiety Disorders, 26, 197 - 205. doi: 10.1016/j.janxdis.2011.11.001

Mattick, R. P. \& Clarke, J. C. (1998). Development and validation of measures of social phobia scrutiny fear and social interaction anxiety. Behaviour research and therapy, 36(4), 455-470. doi:10.1016/ S0005-7967(97)10031-6

McLean, C. P., Asnaani, A., Litz, B. T. \& Hofmann, S. G. (2011). Gender differences in anxiety disorders: Prevalence, course of illness, comorbidity and burden of illness. Journal of Psychiatric Research, 45, 1027-1035. doi: 10.1016/j.jpsychires.2011.03.006

Moral, J., García, C. \& Antona, C. (2013) Validación de la escala de ansiedad en la interacción social en estudiantes universitarios mexicanos. Pensamiento Psicológico, 11, 27 - 42.

Montesi, J., Conner, B., Gordon, E., Fauber, R., Kim, K. \& Heimberg, R. (2013) On the Relationship Among Social Anxiety, Intimacy, Sexual Communication, and Sexual Satisfaction in Young Couples. Archives of Sex Behavior, 42, 81 - 91. doi: 10.1007/s10508-012-9929-3

Ortiz-Gómez, M. \& Muñoz-García, A. (2005). El uso desadaptativo de internet en la adolescencia/juventud. International Journal of developmental and Educational Psychology, 1, 429 - 442.

Orzack, M. H. \& Ross, C. J. (2000). Should virtual sex be treated like other sex addictions?. Sexual Addiction \& Compulsivity: The Journal of Treatment and Prevention, 7(1-2), 113-125. doi: https://doi. org/10.1080/10720160008400210

Panayides, P. \& Walker, M. J. (2012). Evaluation of the Psychometric Properties of the Internet Addiction Test (IAT) in a Sample of Cypriot High School Students: The Rasch Measurement Perspective. Europe's Journal of Psychology, 8, 327-351.doi: 10.5964/ejop.v8i3.474

Pavot, W. \& Diener, E. (2008). The Satisfaction With Life Scale and the emerging construct of life satisfaction. Journal of Positive Psychology, 3, 137-152. doi: 10.1080/17439760701756946

Philaretou, A., Mahfouz, A. \& Allen, K. (2005) Use of Internet Pornography and Men's Well-Being. International Journal of Men's Health, 4, 149-169.

Roberts, J., Hodgson, R. \& Dolan, P. (2011). “It’s driving her mad": Gender differences in the effects of commuting on psychological health. Journal of Health Economics, 30, 1064-76. doi: 10.1016/j.jhealeco.2011.07.006

Rosenfield, S. \& Mouzon, D. (2012). Gender and Mental Health. En: Handbook of The Sociology of Mental Health, 277-296.

Schiebener, J., Laier, C. \& Brand, M. (2015). Getting stuck with pornography? Overuse or neglect of cybersex cues in a multitasking situation is related to symptoms of cybersex addiction. Journal of behavioral addictions, 4(1), 14-21. doi: 10.1556/JBA.4.2015.1.5

Schlenker, B. R. \& Leary, M. R. (1982). Social anxiety and self-presentation: A conceptualization model. Psychological Bulletin, 92, 641-669.

Sears, D. O. (1967). Social anxiety, opinion structure, and opinion change. Journal of Personality and Social Psychology, 7(2p1), 142.

Starcevic, V. \& Aboujaoude, E. (2017). Internet addiction: reappraisal of an increasingly inadequate concept. Cns Spectrums, 22, 7-13. doi: 10.1017/S1092852915000863

Stewart, S. H., Taylor, S. \& Baker, J. M. (1997). Gender differences in dimensions of anxiety sensitivity. Journal of Anxiety Disorders, 11, 179 - 200. doi: 10.1016/S0887-6185(97)00005-4

Storms, M. D. (1980). Theories of sexual orientation. Journal of Personality and Social Psychology, 38(5), 783-792. doi:10.1037/0022-3514.38.5.783

Tan, K.-A. (2018). The Effects of Personal Susceptibility and Social Support on Internet Addiction: an Application of Adler's Theory of Individual Psychology. International Journal of Mental Health and Addiction, 1. 1 - 11 doi: doi.org/10.1007/s11469-018-9871-2

Tylka, T. (2015). No Harm in Looking, Right? Men’s Pornography Consumption, Body Image, and Well-Being. Psychology of Men \& Masculinity, 16, 97 - 107. doi: 10.1037/a0035774

Vázquez, C., Hervás, G., Rahona, J., y Gómez, D. (2009). Bienestar Psicológico y salud: aportaciones desde la psicología positiva. Anuario de Psicología Clinica y de la salud, 5, 15 - 28.

Watson, D. \& Friend, R. (1969). Measurement of social-evaluative anxiety. Journal of consulting and clinical psychology, 33(4), 448. doi: $10.1037 / \mathrm{h} 0027806$

Wéry, A., \& Billieux, J. (2015). Problematic cybersex: Conceptualization, assessment, and treatment. Addictive Behaviors, 64, 238-246. 